\title{
LETTER \\ Simple Oblivious Routing Method to Balance Load in Network-on-Chip
}

\author{
Jiao GUAN ${ }^{\dagger}$, Jueping CAI ${ }^{\dagger a}$, Ruilian $\mathrm{XIE}^{\dagger \dagger}$, Yequn WANG ${ }^{\dagger \dagger \dagger}$, Nonmembers, and Jinzhi LAI ${ }^{\dagger}$, Member $^{\circ}$
}

SUMMARY This letter presents an oblivious and load-balanced routing (OLBR) method without virtual channels for 2D mesh Network-on-chip (NoC). To balance the traffic load of network and avoid deadlock, OLBR divides network nodes into two regions, one region contains the nodes of east and west sides of $\mathrm{NoC}$, in which packets are routed by odd-even turn rule with $\mathrm{Y}$ direction preference $(\mathrm{OE}-\mathrm{YX})$, and the remaining nodes are divided to the other region, in which packets are routed by odd-even turn rule with alterable priority arbitration (OE-APA). Simulation results show that OLBR's saturation throughput can be improved than related works by $11.73 \%$ and OLBR balances the traffic load over entire network.

key words: network-on-chip (NoC), load balancing, oblivious routing

\section{Introduction}

Network-on-chip (NoC) has been proposed as an enormously scalable solution to address the communication demands of Multi-/Manycore systems due to its reusability, scalability, and parallelism in communication infrastructure [1]. Routing method influences the performance of NoC significantly, which determines a path from a source node to destination node [2]. Routing methods of NoC are divided into deterministic routing methods, oblivious routing methods and adaptive routing methods. The deterministic routing methods always choose the same path for communication between a source and a destination. Hence, it has the advantage of being easy to implement and easy to avoid deadlock. However, with the increase of injection rate, single path and large number of packets, network congestion is easy to result in the increase of packet latency and the rapid decline of network performance. According to the network congestion status, the adaptive routing methods can select the uncongested output link to route packets, which guarantee the maximum of network performance. However, the extra overhead of information transmission is introduced, and routers are complicated to avoid deadlock and livelock.

The oblivious routing methods can route packets through any of multiple candidate paths between a pair of

Manuscript received January 17, 2021.

Manuscript revised May 8, 2021.

Manuscript publicized June 30, 2021.

${ }^{\dagger}$ The authors are with State Key Laboratory of Wide Bandgap Semiconductor Technology Disciplines, Xidian University, Xi'an, 710071, China.

${ }^{\dagger}$ The author is with Institute of Computer Science \& Technology, Xi' an University of Posts \& Telecommunications, Xi' an 710061, China.

${ }^{\dagger \dagger}$ The author is with Institude of Information and Navigation, Air Force Engineering University, Xi' an, 710077, China.

a)E-mail: jpcai@mail.xidian.edu.cn

DOI: 10.1587/transinf.2021EDL8005 nodes A and B without consider the network status, which has a relatively good balance between locality and load balance. For example, O1TURN [3] is a well-known oblivious routing method that has been shown to be worst-case throughput optimal for even network radices, but not for odd radices. U2TURN [4] improves over the O1TURN method. This is accomplished by load balancing among 2-turn paths in XYX or YXY routing. U2TURN achieves higher throughput than O1TURN under adversarial traffic. However, for random traffic, where the traffic is inherently load-balanced, O1TURN achieves lower network latency since it only considers minimal paths. UMF [5] combines the advantages of O1TURN and U2TURN into one oblivious routing method. When the traffic is already inherently load-balanced, UMF employs O1TURN routing rules to transform packets. When a load-imbalance is detected, UMF switches to U2TURN routing rules to transform packets. However, the UMF need virtual channel to avoid deadlock.

The oblivious routing methods are simple. However, the central nodes of $\mathrm{NoC}$ with oblivious routing are about $2 \times$ more congested in comparison with lateral nodes. To balance the load of the central nodes, LAXY [6] routing method distribute packets over the lateral nodes, in which the certain routers at the east and the west of network are configured as $\mathrm{YX}$ routing, the other routers are configured as XY. As depicted in Fig. 1, LAXY transfers the packets of central nodes (red solid arrows) through less congested lateral nodes (blue dotted arrows). However, the LAXY cannot balance the partial traffic load and need a deadlock recovery technique to deal with the happened deadlock.

The Odd-Even (OE) turn model [7] is proposed as a maximally adaptive routing method without virtual channels. As the odd columns and even columns appear alternatively in the network, conventional OE turn model is limited to a relatively determined routing path. To improve the

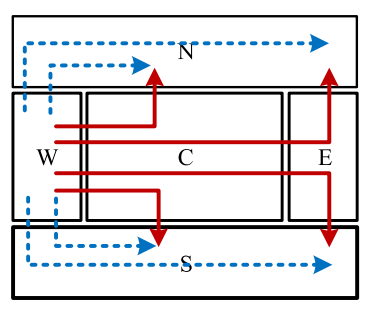

Fig. 1 LAXY routing method. 
number of candidate paths of OE turn model, OE-Arbiter [8] proposes an alterable priority arbitration (APA) for the fair granting of packet transmission from all possible directions permitted by the OE turn model. By applying the APA as selection for permitted routing directions, more candidate paths are provided to relieve congestion, the packet latency is reduced and the network capacity is improved.

In this letter, we propose an Oblivious and LoadBalanced Routing (OLBR) method without virtual channels for 2D mesh NoC. OLBR statically divides NoC nodes into two regions: one region contains the nodes at the east and the west of network; the remaining nodes are divided to the other region. To reduce the traffic load of the central nodes of network and avoid deadlock, OLBR employs different OE routing rule for the two regions.

\section{Region Partitioning Model}

In this letter, we adopt the Fishtail shape region model [6] for the analysis and simulation. The difference of region size and shape will affect the distribution of network traffic. In Fishtail configuration, the outer side of the regions west and east is wider than the inner side and narrows down toward the center, which reduces the number of routers in the central region comparison with east and west sides. These regions can be categorized into two classes: East-West (WE) region and North-South (NS) region, as shown in Fig. 2.

\section{Proposed Routing Method}

In this section, based on Fishtail shape region model, the $\mathrm{NoC}$ is dived into two regions. In WE region, packets are routed by $\mathrm{OE}$ turn rule with $\mathrm{Y}$ direction preference, which diverts the traffic through central node to the north and south. When the traffic from the central node is transferred to the NS region, this region will become congested. In NS region, to improve the number of candidate output path of the conventional OE routing method, OLBR router employs the Alterable Priority Arbitration (APA) [8] for the fair granting of packet transmission from all possible directions permitted by the OE turn model. APA applies the granting mechanism of FP arbiter under varied priorities instead of fixed order, while the pointer to adjust the priority order is optimized for alterable priority arbitration.

Packets are routed inside the region using the following two rules. The $\mathrm{C}, \mathrm{S}$ and $\mathrm{D}$ represent current node, source

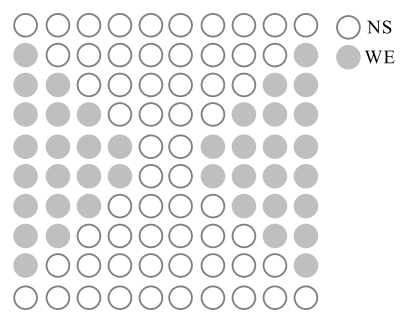

Fig. 2 Region partitioning model. node and destination node respectively.

Rule 1: WE Region routing.

When $\mathrm{C}$ is in the WE region, packets are routed by OE-YX routing method. If $\mathrm{C}$ is in an even (odd) column and $\mathrm{D}$ is at the northwest (southwest) side of $\mathrm{C}$, packets are first routed West to the odd (even) column. Otherwise, the packets are routed by the $\mathrm{Y}$ direction firstly and $\mathrm{X}$ direction secondly (YX) routing. Since the packets of central nodes are transferred through the nodes at the east and west side of network, the load of central nodes are balanced. Since the packets of central nodes are transferred through the nodes at the east and west side of network, the load of central nodes are balanced.

Rule 2: NS Region routing.

When $\mathrm{C}$ is in the NS region, packets are routed using OE-APA routing method. If $\mathrm{D}$ is at the northwest or southwest side of C, packets are forbidden to route toward north and south directions in odd columns to avoid NW and SW turns. The packets are not restricted in even columns, in which APA is employed to select an output port of all shortest path. If $\mathrm{D}$ is at the northeast or southeast side of $\mathrm{C}$, packets are forbidden to route north and south directions on even columns to avoid EN and ES turns. The packets are not restricted in odd columns, in which APA is employed to select an output port of all shortest path. OE-APA improves the number of candidate output path and ensures the load balancing within the region.

A special case is when D is in even column, in which the eastbound packets are routed toward $\mathrm{Y}$ direction at $\mathrm{X}_{\mathrm{D}-1}$ column to avoid EN and ES turns in the $\mathrm{X}_{\mathrm{D}}$ column.

In Fig. 3, we use two examples to show how LAXY, OE-Arbiter and OLBR route packets in a $8 \times 8$ mesh NoC. Packets, source node $(1,0) \rightarrow$ destination node $(5,5)$, are routed toward northeast direction and the source node is in NS region. According to Rule 2, no limitation for directions exists on odd column, arbitration for $\mathrm{E}$ direction and $\mathrm{N}$ direction is required. At even column, $\mathrm{N}$ direction is prohibited, no arbitration is required, so the packets are routed in $\mathrm{E}$ direction. This process is repeated until the packets are routed to node $(5,3)$. The node $(5,3)$ is in the WE region, so the packets are then routed to the destination node by Rule 1 . These three algorithms route all of the packets

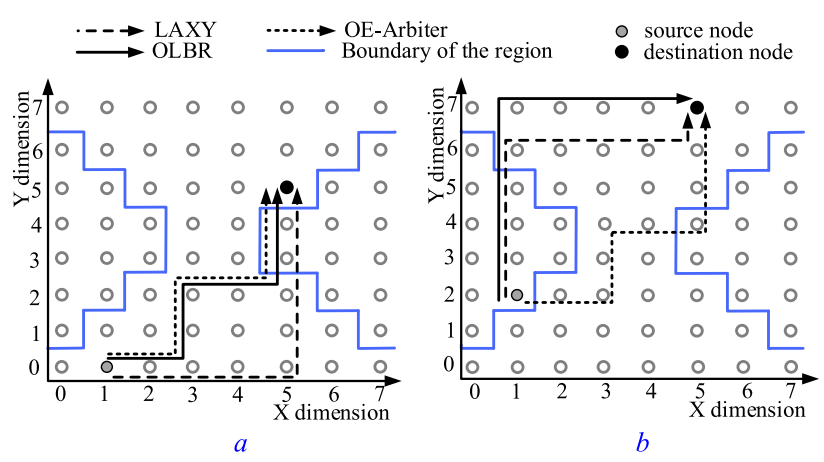

Fig. 3 Two routing examples in an $8 \times 8$ mesh. (a) The source node is in NS region, (b) the source node is in WE region. 
through the shortest path. Different from OE-Arbiter and LAXY limited to relatively fixed routing path, OLBR transfers packets along the east and west side of network when the source node is in the WE region. When the source node is in the NS region, OLBR provide more candidate paths for transferred packets.

\section{Simulation Results}

We introduce two methods: (1) static analysis and (2) dynamic simulation, to evaluate the perform of these OE [7], OE-Arbiter [8], LAXY [6] and OLBR.

\subsection{Static Analysis}

Statistical traffic load distribution (STLD) [9] helps us to predict the traffic load of the network statistically without real simulations. According to different routing methods, the STLD collects all source-destination pairs to estimate real simulations with uniform distribution. Let the packets of all source-destination pairs route once and statistically count the number of packets passing through in each router. We assume that the network topology size is $8 \times 82 \mathrm{D}$ mesh, and adopt the Fishtail shape region model which have been described in Sect. 2.

The STLDs of OE-Arbiter [8], LAXY [6] and OLBR are shown in Fig. 3. The color bar represents the range of the statistical traffic loads and the bold black lines mean the boundaries of the region. For LAXY, the routers in WE region are configured as YX routing and the routers in NS region are configured as XY routing. For OLBR, the routers in WE region are configured as OE-YX and the routers in NS region are configured as OE-APA.

As shown in the STLD graph in Fig. 4(a), the most congested routers at the center of the network is about $1.7 \times$ more congested than average traffic of the lateral routers. For the STLD of LAXY in Fig. 4(b), the traffic of central routers is reduced by $56 \%$ over OE-Arbiter. This is because packets from the WE region are routed south and north, not through the central router. However, the traffic load on the inner region boundary routers becomes more congested, which diminishes the effectiveness of LAXY. In contrast, the OLBR results in balanced statistical traffic loads not only in the center of the network but also on the whole network in Fig. 4 (c). This is because OE-APA significantly improves the number of candidate routing paths, more possible paths are provided. The simulation results show OLBR utilizes

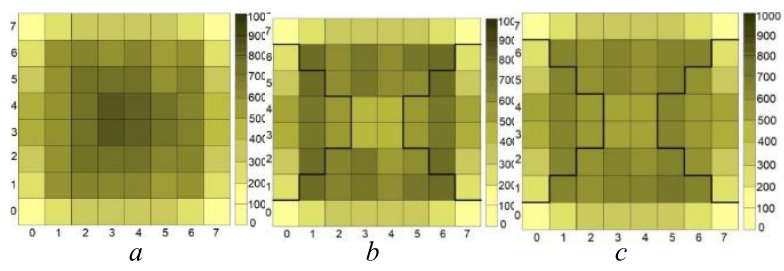

Fig. 4 Statistical traffic load distributions of (a) OE-Arbiter [8], (b) LAXY [6] and (c) OLBR. the network routers more uniform than the other routing methods.

\subsection{Dynamic Simulation}

We use BookSim2.0 [10] to evaluate OLBR in average network latency with OE [8], OE-Arbiter [8] and LAXY [6]. We evaluate the four routing methods using four standard traffic patterns: uniform traffic, transpose 1 traffic, transpose 2 traffic and hotspot traffic. Under transpose traffic pattern, the coordinates of destination node are determined by transposing the source node coordinates based on determined rules: for $\mathrm{X} \times \mathrm{Y}$ mesh, packets on node $(\mathrm{i}, \mathrm{j})$ are delivered to node $(X-1-j, Y-1-i)$ under transpose 1 and to node $(\mathrm{j}, \mathrm{i})$ under transpose2. Hotspot traffic pattern is based on the uniform traffic and the hotspot node are $(4,4)$. The probabilities of sending packet to hotspot node are 5\%. We assume that the network topology size is $8 \times 82 \mathrm{D}$ mesh, and adopt the Fishtail shape region model which have been described in Sect. 2. The packet size is randomly selected between 2 and 5 flits. Two adjacent nodes are connected by two unidirectional channels, and each channel has eight flit buffers. The simulator is warmed up for 10,000 cycles, and then, the average performance is measured over another 40,000 cycles. The average latency under different packet injection rates is used as the performance index of the simulations. We also adopt the saturation throughput [11], which is the throughput where the average latency equals to twice of the zero-load latency, as the evaluation metric.

The simulation results are presented in Fig. 5. For uniform traffic pattern, the performance of the four routing methods is similar. This is because the traffic is inherently load-balanced when the destinations are chosen at uniform random. OE-Arbiter is slightly better than LAXY, due to LAXY requires an additional deadlock recovery mechanism to avoid deadlock. For the other three traffic patterns, the
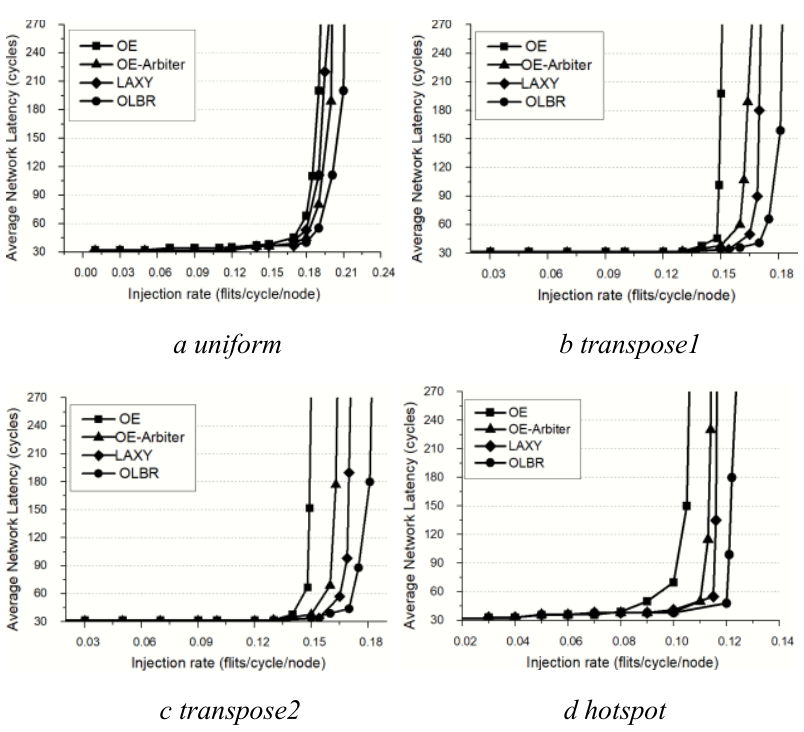

Fig. 5 Performance analysis. 
saturation throughput of OE-Arbiter is higher than OE. This is because the OE-Arbiter significantly improves the number of candidate routing paths, more possible paths are provided for packets, which reduce the time wasted on waiting in congested ports. The simulation results show LAXY makes the network traffic of nodes more balanced than OEArbiter except uniform traffic pattern. This is because the network traffic is focused at the center of the network, and LAXY removes their traffic from the central nodes to the north and south regions of network. OLBR combines the advantages of LAXY and OE-Arbiter into one oblivious routing method. Compare with OE-Arbiter, OLBR can improve the saturation throughput by $5.12 \%$ under uniform pattern, $11.73 \%$ under transpose 1 pattern, $8.85 \%$ under transpose 2 pattern and $9.73 \%$ under hotspot patterns respectively. Besides, compared with LAXY, OLBR improves the saturation throughput by $5.9 \%, 7.5 \%, 7.31 \%$ and $6.03 \%$ for uniform pattern, transpose 1 pattern, transpose 2 pattern and hotspot patterns, respectively.

\section{Conclusion}

In order to balance the load of the oblivious routing method, OLBR routing method is proposed. OLBR divides nodes into two regions, one region contains the nodes of east and west sides of network, in which packets are routed by oddeven turn rule with $\mathrm{Y}$ direction preference (OE-YX), and the remaining nodes are divided to the other region, in which packets are routed by odd-even turn rule with alterable priority arbitration (OE-APA). That is, OLBR combines the advantages of LAXY and OE-Arbiter into one oblivious routing method. Simulation results demonstrate that OLBR, no matter how the traffic of load is changed, can provide a better performance in reducing the saturation throughput than state-of-the-art oblivious routing methods designed for $\mathrm{NoC}$ without virtual channels. Meanwhile, OLBR utilizes the network nodes more uniform than their routing methods.

\section{Acknowledgments}

This work is supported by the innovation wisdom base for wide bandgap semiconductor and micro-nano electronics of China (B12026). The key projects in strategic international scientific and technological innovation cooperation (2016YFE0207000).

\section{References}

[1] S. Hesham, D. Goehringer, and M.A. Abd El Ghany, "HPPT-NoC: A dark-silicon inspired hierarchical TDM NoC with efficient powerperformance trading," IEEE Trans. Parallel Distrib. Syst., vol.31, no.3, pp.675-694, 2020.

[2] A. Mehranzadeh, A. Khademzadeh, N. Bagherzadeh, and M. Reshadi, "DICA: Destination intensity and congestion-aware output selection strategy for network-on-chip systems," IET Comput. Digit. Tech., vol.13, no.4, pp.335-347, 2019.

[3] D. Seo, A. Ali, W.-T. Lim, N. Rafique, and M. Thottethodi, "Near-optimal worst-case throughput routing for two-dimensional mesh networks," ACM SIGARCH Computer Architecture News, vol.33, no.2, pp.432-443, 2005.

[4] G. Sun, C.-W. Chang, and B. Lin, "A new worst-case throughput bound for oblivious routing in odd radix mesh network," IEEE Comput. Archit. Lett., vol.12, no.1, pp.9-12, 2013.

[5] Y. Song and B. Lin, "Uniform minimal first: Latency reduction in throughput-optimal oblivious routing for mesh-based networkson-chip," IEEE Embed. Syst. Lett., vol.11, no.3, pp.81-84, 2019.

[6] N. Rohbani, Z. Shirmohammadi, M. Zare, and S.-G. Miremadi, "LAXY: A location-based aging-resilient Xy-Yx routing algorithm for network on chip," IEEE Trans. Comput.-Aided Des. Integr. Circuits Syst., vol.36, no.10, pp.1725-1738, 2017.

[7] G.-M. Chiu, "The odd-even turn model for adaptive routing," IEEE Trans. Parallel Distrib. Syst., vol.11, no.7, pp.729-738, 2000.

[8] L. Liu, Z. Zhu, D. Zhou, and Y. Yang, "A fair arbitration for networkon-chip routing with odd-even turn model," Microelectronics Journal, vol.64, pp.1-8, 2017.

[9] S.-Y. Lin, C.-H. Huang, C.-H. Chao, K.-H. Huang, and A.-Y. $\mathrm{Wu}$, "Traffic-balanced routing algorithm for irregular mesh-based on-chip networks," IEEE Trans. Comput., vol.57, no.9, pp.11561168, 2008.

[10] W. Dally and B. Towles, Principles and Practices of Interconnection Networks, Morgan Kaufmann, San Francisco, CA, USA, 2004.

[11] L. Shang, L.-S. Peh, and N.K. Jha, "PowerHerd: A distributed scheme for dynamically satisfying peak-power constraints in interconnection networks," IEEE Trans. Comput.-Aided Des. Integr. Circuits Syst., vol.25, no.1, pp.92-110, 2006. 